\title{
Percepción y conocimiento de los estudiantes universitarios de Administración y Dirección de Empresas sobre el Espacio Europeo de Educación Superior (EEES)
}

\section{Perception and knowledge of the business administration university students about the European Higher Education Area}

\author{
Manuel EXPÓSITO-LANGA, José-Vicente TOMÁS-MIQUEL y \\ Gema TORRES-GALLARDO \\ Universitat Politècnica de València
}

Recibido: Febrero 2012

Aceptado: Abril 2012

\section{Resumen}

La Declaración de la Sorbona fue un paso fundamental de un proceso político de construcción de un Espacio Europeo de Educación Superior. No cabe duda que esta reforma tiene un efecto directo sobre toda la comunidad universitaria y acontece cambios que en ocasiones desconciertan a todos. En este contexto, nos planteamos como objetivos del trabajo comprobar qué saben y qué opinan los alumnos universitarios sobre este proceso. Para responder a estos objetivos hemos trabajado con alumnos matriculados en los estudios de Administración y Dirección de Empresas del Campus de Alcoy de la Universitat Politècnica de València. En cuanto a los resultados, observamos que un $76 \%$ de los alumnos sí aprueba una prueba objetiva de conocimientos, si bien la nota media obtenida ha sido de 5,58, valor relativamente bajo. Diferenciando por cursos, los alumnos de primero son lo que mejor calificación han obtenido respecto al resto de cursos, por otro lado, los alumnos de licenciatura, en general, presentan una opinión más crítica respecto al proceso de implantación del Espacio Europeo de Educación Superior. Los resultados del trabajo implican la necesidad de reajustar las políticas de difusión y comunicación, ya que así, consideramos que el Espacio Europeo de Educación Superior tendría una implantación más efectiva.

Palabras clave: Espacio Europeo de Educación Superior, Plan Bolonia, Administración y Dirección de Empresas, Enseñanzas Universitarias, Estudiantes Universitarios.

\begin{abstract}
The Sorbonne Declaration was a major step in a political process of building a European Higher Education Area. There is no doubt that this education reform has a direct effect on the entire university community and involves changes that often confound everyone. In this context, we
\end{abstract}


consider the objectives of this work to study what university students know and think about everything concerning this process. To meet these objectives, we have worked with students enrolled in the studies of Business Administration of the Campus of Alcoy of the Universitat Politècnica de València. Regarding the results, we observed that $76 \%$ of the students pass the knowledge test and a relatively low final average mark of 5.58. Differentiating by courses, first course students have achieved the best marks compared to other courses; otherwise, students of last courses, which are still based on the old configuration of degree studies, generally have a more critical view of the process of implementation of the European Higher Education Area. The results of this work, in our opinion, imply the need to readjust the distribution and communication policies in order to achieve a more effective implementation of the European Higher Education Area.

Keywords: European Higher Education Area, Bologna Process, Business Administration, Higher Education teaching, University students.

\section{El modelo de Convergencia Europea}

El modelo de Convergencia Europea tiene sus orígenes en el 25 de mayo de 1988, cuando los ministros de Educación de Francia, Alemania, Italia y Reino Unido firman en la Sorbona una declaración que insta al desarrollo de un Espacio Europeo de Educación Superior (EEES). Sin duda, esto fue la primera piedra de un largo proceso de cambio de la enseñanza superior en Europa.

Posteriormente, una nueva conferencia en junio de 1999 dio lugar a la Declaración de Bolonia, suscrita ya por 30 estados de Europa, y donde se sentaron las bases para la construcción del EEES partiendo de los principios de calidad, movilidad, diversidad y competitividad, tanto para el alumnado como para el profesorado. En la Declaración de Bolonia (1999) se presentaba un conjunto de objetivos e instrumentos para el logro, y establecía como plazo 2010 para consolidar el EEES. Una detallada reflexión sobre la incorporación de las universidades al EEES se puede consultar en Alba Pastor (2005).

Durante este proceso han tenido lugar múltiples reuniones (con carácter bianual) para poner en común avances, llevar a cabo un seguimiento general e incorporar nuevos países miembros al acuerdo.

Haciendo un breve recorrido por estas reuniones, en 2001 en Salamanca se realizaron trabajos previos a la Conferencia de Ministros responsables de la educación superior que posteriormente tuvo lugar en Praga, siendo ésta la primera conferencia de seguimiento. En Barcelona en 2002 se desarrolló la Cumbre de Jefes de Estado donde establecieron las bases para garantizar la movilidad y el reconocimiento profesional. En 2003 tuvo lugar la $2^{\mathrm{a}}$ Convención de instituciones europeas de Graz, y la reunión de Ministros de Berlín, donde se puso en relevancia la función e importancia de las redes y organismos de evaluación de la calidad del EEES. Posteriormente, en 2005, la Declaración de Glasgow fijó como prioridad en Europa el futuro de sus universidades. De nuevo en 2005, el Comunicado de Bergen recopiló los hitos alcanzados durante todo el proceso. En 2007 se celebró en Londres una nueva Conferencia Ministerial en la que se adoptó el EEES dentro de un escenario global. Por último, y ya en 2009 hubo otra Conferencia Ministerial en Benelux donde se marcaron nuevas orientaciones políticas para los próximos años. 


\section{El Espacio Europeo de Educación Superior}

En la Declaración de la Sorbona (1998) se puso el acento en la función de la universidad en el desarrollo de una "Europa del conocimiento", mucho más allá que una "Europa del Euro". Consolidar las dimensiones intelectuales, culturales, sociales y técnicas es clave para el desarrollo del continente, y es obvio el papel que desempeñan las universidades en ese proceso. De esta forma, disponer de un modelo europeo de universidad con criterio, reflexión y recursos, capaz de preparar profesionales para desenvolverse en un contexto cada vez con mayores exigencias competitivas tanto técnicas y científicas como humanas, en una Europa abierta, con respeto a la diversidad, que favorezca la movilidad de estudiantes y profesorado y la cooperación, es sin duda un reto que requiere un gran esfuerzo colectivo y de carácter continuo. Este fin, llevará al establecimiento de un marco común de referencia que permita una verdadera convalidación europea de aquellos conocimientos y destrezas adquiridas. En definitiva todas estas ideas se desarrollan en base a:

- Adoptar un sistema comparable de títulos y la expedición del suplemento europeo al título para promover las oportunidades de trabajo y la competitividad del sistema de enseñanza superior a escala internacional.

- La adopción de una estructura de estudios basada en dos ciclos, grado y postgrado.

- La promoción de la movilidad de estudiantes, investigadores y profesores en el contexto europeo.

- La promoción de la cooperación europea en materia de garantía de calidad para el desarrollo de criterios y metodologías educativas comparables.

- La promoción de la dimensión europea en la educación superior, sobre todo en lo referente a la elaboración de planes de estudios, cooperación interinstitucional, programas de movilidad y programas integrados de estudios, formación e investigación.

En resumen, estamos ante un proceso que viene de lejos y cuyos esfuerzos se están materializando recientemente con la implantación de los nuevos grados y la desaparición progresiva de las ya antiguas titulaciones. Desde el mundo universitario se ha intentando transmitir a través de diferentes medios como Internet (webs y foros), informes, revistas, folletos, etc. la importancia de estos cambios y las nuevas metodologías y exigencias a los alumnos, principales destinatarios de todas estas acciones, que ven arrancar una nueva operativa y conceptualización de su proceso formativo.

Sin duda, estamos ante un periodo de cambio educativo, donde la adaptación al nuevo sistema no es algo trivial ni automático de alguna asignatura, sino que está impulsando un importante movimiento de renovación pedagógica en la Educación Superior Española (Florido de la Nuez et al., 2008) que hay que aprovechar para poner en marcha nuevas metodologías, y donde el rol del profesor y el del alumno son clave para que el resultado del proceso de enseñanza-educativo sea significativo (Díez et al., 2009). Por este motivo, pensamos que la aplicación del EEES no será efectiva a menos que los alumnos posean un conocimiento completo de lo que significa este nuevo proceso, así como de sus principales características y metodologías docentes, y sean partícipes con una mayor presencia de sus opiniones en el proceso de implantación (Ocaña 
y Reyes, 2011). De esta forma, nos planteamos como objetivos de este estudio evaluar el nivel de conocimientos generales, así como la percepción que los alumnos tienen sobre las implicaciones de la implantación del EEES.

Para nuestro estudio empírico hemos seleccionado, por proximidad, a los alumnos de la disciplina de Administración y Dirección de Empresas del Campus de Alcoy de la Universitat Politècnica de València.

Una vez identificado el contexto y objetivo de nuestro trabajo, a continuación desarrollaremos el estudio empírico llevado a cabo, los principales resultados obtenidos y finalizaremos con unas conclusiones o reflexiones.

\section{Estudio empírico}

El Campus de Alcoy, con un total de 2.327 alumnos (curso 2011-2012), es uno de los campus externos que tiene la Universitat Politècnica de València. Alcoy es una ciudad situada en la provincia de Alicante que cuenta con una población aproximada de 65.000 habitantes y una tradición en la enseñanza superior, principalmente en el campo de la ingeniería, de más de 150 años de experiencia. En el curso 2010-2011, y siguiendo el plan estratégico establecido por la propia Universitat Politècnica de València, se han implantado los primeros cursos de un total de 6 grados, que conviven con las 11 titulaciones existentes de primer y segundo ciclo y que se irán extinguiendo de forma progresiva.

Entre estas titulaciones, se encuentra Administración y Dirección de Empresas como una de las que más aceptación tiene en cuanto a matrícula, siendo 377 alumnos matriculados en el curso 2010-2011 entre el recién implantado primer curso del grado y los que restan de la licenciatura a extinguir, es decir de segundo a quinto.

En estos estudios se pretende capacitar al alumno para que pueda desarrollar su actividad con desenvoltura en un contexto de gerencia o de dirección de las diferentes áreas específicas de una organización, tanto privada como pública (comercialización, recursos humanos, finanzas, etc.), así como tareas de consultoría y asesoría a terceros.

Esta motivación compartida tanto por los alumnos, como por el profesorado y el personal de administración nos conduce a la búsqueda de una diferenciación con otras implantaciones del mismo título en el mapa de universidades tanto valenciano como español. Así, valores como la proximidad al alumno y el esfuerzo continuo y compartido, hacen que sea atractiva y motivadora para el alumno, dando lugar a profesionales con una formación humana integral.

\section{Confección de la muestra y fuentes de datos}

Para la confección de la muestra de nuestro estudio hemos considerado la totalidad de alumnos del Campus de Alcoy de la Universitat Politècnica de València matriculados en los cursos de grado y licenciatura en Administración de Empresas. El trabajo de campo abarcó el mes de febrero de 2011 y la fuente de información básica fue la apor- 
tada por las respuestas a una prueba con preguntas sobre el Plan Bolonia (ver Anexo I). La prueba estaba dividida en dos bloques, uno asociado a una prueba objetiva de conocimientos (10 ítems de opción múltiple) y otro asociado a una prueba de percepción (16 preguntas con valoración en escala Likert 1-5) sobre el EEES.

Para pasar la prueba pedimos la colaboración de un profesor de cada uno de los cursos que impartiera alguna asignatura troncal, con el objeto de obtener la máxima participación posible del alumnado. Al principio de la clase se repartió la prueba, que tenía una duración prevista de unos 10 minutos. Previamente se transmitió a los alumnos el objetivo de la investigación y se les pidió la máxima sinceridad en las respuestas. Cabe apuntar que anteriormente llevamos a cabo una prueba piloto con un grupo reducido de alumnos y un par de profesores con el objeto de ajustar y confirmar que las preguntas eran comprensibles y estaban bien formuladas.

Este trabajo de campo dio como resultado un total de 221 respuestas válidas sobre un total posible de 377 alumnos matriculados. El resultado supone un nivel de respuesta del $58,6 \%$ y un error muestral del $4,33 \%$, valores que se consideran adecuados para poder establecer un análisis posterior. La Tabla 1 muestra la ficha técnica del estudio.

Tabla 1. Ficha técnica del estudio

\begin{tabular}{|c|c|}
\hline UNIVERSO & $\begin{array}{l}\text { Titulación de ADE del Campus de Alcoy de la Univer- } \\
\text { sitat Politècnica de València }\end{array}$ \\
\hline ÁMBITO GEOGRÁFICO & Alcoy (Alicante) \\
\hline MARCO MUESTRAL & $\begin{array}{l}\text { Todos los alumnos matriculados en cualquiera de los } 5 \\
\text { cursos académicos (total población } 377 \text { alumnos) }\end{array}$ \\
\hline ELEMENTO MUESTRAL & Alumnos \\
\hline TAMAÑO MUESTRAL & 221 respuestas válidas \\
\hline ERROR MUESTRAL & $\begin{array}{l} \pm 4,33 \% \text { (Nivel de confianza del } 95,5 \%, z=1,96 \\
p=q=0,5)\end{array}$ \\
\hline DISEÑO MUESTRAL & Diseño y Pre-test del cuestionario \\
\hline INFORMACION RECOGIDA & $\begin{array}{l}\text { Conocimiento y percepción del Plan Bolonia en cuanto } \\
\text { a objetivos y puesta en marcha, estructura, metodolo- } \\
\text { gía, homologación y financiación }\end{array}$ \\
\hline
\end{tabular}

\section{Resultados}

\section{Análisis de la prueba de conocimiento}

A continuación mostramos algunos estadísticos descriptivos de los resultados obtenidos en la primera parte de la prueba. Las preguntas de la prueba objetiva de conocimiento están agrupadas en 5 áreas de interés o categorías: Objetivos y puesta en marcha, Estructura, Metodología, Homologación y Financiación del Plan Bolonia. En la Tabla 2 hemos calculado la nota media obtenida por los alumnos tanto de forma global como para cada una de las áreas. Como se puede observar, el conjunto de los alumnos aprueban (media $=5,58$ ), si bien se puede interpretar que la nota no es muy alta, por lo que, aunque sí conocen el Plan Bolonia, dicho conocimiento no es el deseable para un 
modelo que les afecta plenamente. Este resultado concuerda con otros trabajos (García Ríos et al., 2006, Díez et al., 2009) donde se pone de manifiesto que el desconocimiento de la organización del grado es una circunstancia relevante que da lugar a problemas de diversa índole. Por otro lado, si analizamos la nota media obtenida por cada área, parece que sí conocen bien las nuevas metodologías que se pretenden incorporar (por ejemplo, la evaluación continua), así como la estructura en créditos ECTS y la homologación de título a nivel europeo. Sin embargo, suspenden en el conocimiento relativo a los objetivos básicos del Plan y a las modalidades de financiación de préstamos rentauniversidad.

Tabla 2. Media y desviación típica de la prueba de conocimiento por área (subgrupos de preguntas) y media del global de preguntas de la prueba objetiva

\begin{tabular}{|l|c|c|}
\hline & Media & Desviación Típica \\
\hline $\begin{array}{l}\text { Objetivos y puesta en } \\
\text { marcha }\end{array}$ & 3,10 & 3,30 \\
\hline Estructura & 5,91 & 2,78 \\
\hline Metodología & 7,94 & 2,93 \\
\hline Homologación & 6,07 & 2,97 \\
\hline Financiación & 3,80 & 4,86 \\
\hline Nota total & 5,58 & 1,59 \\
\hline
\end{tabular}

$\mathrm{N}=221$

Adicionalmente, y con el objeto de enriquecer nuestro trabajo hemos calculado el valor de la media de conocimiento de los alumnos separados por curso. Como se observa en la Tabla 3, los alumnos de primer curso son los que obtienen mejor calificación, lo que es justificable debido a que ya están inmersos en el plan Bolonia. A medida que avanzamos en el resto de cursos (ya de licenciatura), la nota decrece. Este resultado tiene sentido ya que estos alumnos ven de cerca los cambios que están aconteciendo, así como las posibles implicaciones que pueden tener sobre ellos, pero todavía no les afectan directamente.

Tabla 3. Media de la prueba por curso

\begin{tabular}{|c|c|c|c|c|}
\hline \multicolumn{5}{|c|}{ NOTA MEDIA POR CURSO } \\
\hline $\begin{array}{c}\text { Primero } \\
\text { de grado }\end{array}$ & $\begin{array}{c}\text { Segundo de } \\
\text { Licenciatura }\end{array}$ & $\begin{array}{c}\text { Tercero de } \\
\text { Licenciatura }\end{array}$ & $\begin{array}{c}\text { Cuarto de } \\
\text { Licenciatura }\end{array}$ & $\begin{array}{c}\text { Quinto de } \\
\text { Licenciatura }\end{array}$ \\
\hline 6,13 & 5,59 & 5,26 & 5,15 & 5,04 \\
\hline
\end{tabular}

Para validar si los resultados de la prueba presentan diferencias significativas entre sí, consideramos que la aplicación de un modelo ANOVA es adecuado a nuestro 
análisis. La técnica ANOVA permite principalmente comparar medias de diferentes grupos. En este caso, el diseño del experimento tendría una variable independiente o factor (Curso) y tomaríamos como variable dependiente, o respuesta, la nota final obtenida en la prueba.

Hemos llevado a cabo esta prueba con el software SPSS v.16, y el resultado ha sido que efectivamente existen diferencias significativas en la nota media obtenida entre los diferentes cursos (estadístico F $=3,83$ y Sig. $=, 005$ ). Sin embargo, el estadístico F del ANOVA únicamente nos proporciona información para contrastar la hipótesis sobre si los diferentes promedios comparados son iguales. En nuestro caso particular, el valor de significatividad obtenido nos lleva a rechazar esta hipótesis, por lo que es interesante precisar dónde están las diferencias entre los diferentes grupos.

Para esto, hemos llevado a cabo un análisis post hoc o comparación a posteriori. Previamente, necesitamos conocer si se puede, o no, asumir la igualdad de las varianzas poblacionales, para lo que aplicaremos el contraste de Levene. El valor del contraste (estadístico de Levene $=, 727)$ y su nivel crítico $($ Sig. $=, 574)$ nos permite aceptar la hipótesis de igualdad de varianzas, por lo que en los estratos definidos por los diferentes cursos, las varianzas no difieren de forma significativa. Esta consideración es importante ya que nos determinará el tipo particular de contraste que podemos llevar a cabo para establecer la comparación post hoc. En este caso hemos utilizado el método de Tukey, comúnmente aceptado en trabajos de investigación. El resultado obtenido se puede observar en la Tabla 4.

Tabla 4. Comparaciones múltiples post hoc. Prueba de Tukey

\begin{tabular}{|c|c|c|c|c|c|c|c|c|c|c|c|c|c|c|}
\hline \multicolumn{2}{|c|}{ Curso } & \multirow{2}{*}{$\begin{array}{c}\begin{array}{l}\text { Dif. de } \\
\text { medias }\end{array} \\
, 54\end{array}$} & \multicolumn{2}{|c|}{ Curso } & \multirow{2}{*}{$\begin{array}{c}\text { Dif. de } \\
\text { medias }\end{array}$} & \multicolumn{2}{|c|}{ Curso } & \multirow{2}{*}{$\begin{array}{c}\begin{array}{l}\text { Dif. de } \\
\text { medias }\end{array} \\
-, 87^{*}\end{array}$} & \multicolumn{2}{|c|}{ Curso } & \multirow{2}{*}{$\begin{array}{l}\text { Dif. de } \\
\text { medias } \\
-, 98^{*}\end{array}$} & \multicolumn{2}{|c|}{ Curso } & \multirow{2}{*}{$\begin{array}{l}\text { Dif. de } \\
\text { medias } \\
-1,09^{*}\end{array}$} \\
\hline 1 & 2 & & 2 & 1 & & 3 & 1 & & 4 & 1 & & 5 & 1 & \\
\hline & 3 &, $87 *$ & & 3 & ,33 & & 2 &,- 33 & & 2 &,- 44 & & 2 &,- 55 \\
\hline & 4 & ,98* & & 4 & ,44 & & 4 & ,11 & & 3 &,- 11 & & 3 &,- 22 \\
\hline & 5 & $1,09 *$ & & 5 &, 55 & & 5 &, 22 & & 5 &, 11 & & 4 &,- 11 \\
\hline
\end{tabular}

$* \mathrm{p}<, 05$

La relación de valores de la Tabla 4 está basada en el grado de similitud de las medias de los diferentes cursos. A raíz de los resultados obtenidos se observa que la media de los alumnos de primer curso de grado es superior y significativamente diferente del resto, excepto de la de los alumnos de segundo curso de licenciatura. De esta forma, primer curso constituiría por sí mismo un grupo homogéneo. Por otro lado, las notas de los alumnos de tercero, cuarto y quinto son las más bajas y no presentan diferencias estadísticamente significativas entre sí, por lo que constituiría un segundo grupo homogéneo. Por último, el resultado obtenido por los alumnos de segundo curso, si bien no presenta diferencias significativas con el resto, establecería un puente entre los dos grupos homogéneos anteriormente obtenidos, por lo que a priori no podemos considerarlo dentro de ninguno de ellos de forma totalmente definida. Con el objeto de clarificar este resultado incluimos la gráfica de medias por curso (Gráfico 1). 
Gráfico 1. Gráfica de notas medias de la prueba objetiva por curso

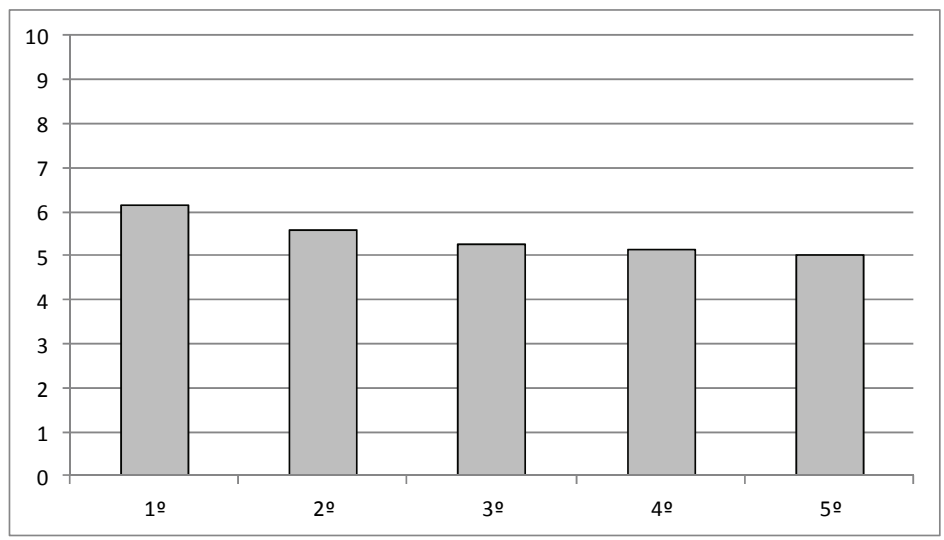

\section{Análisis de la prueba de percepción}

Una vez el alumno completaba la prueba de conocimiento, a continuación respondía a un cuestionario con el objetivo de evaluar cuál es su opinión sobre el EEES. Para llevar a cabo el análisis de esta segunda etapa hemos tenido en cuenta solamente aquéllos que superaban la prueba objetiva de conocimiento con una nota de al menos 5 puntos. Esta forma de actuar es justificable ya que para poder analizar con causa la opinión que los alumnos tienen sobre cualquier aspecto determinado del EEES, es importante que previamente se hayan interesado y conozcan en qué consiste esa realidad. Por otro lado, pensamos que la opinión de aquéllos alumnos que no superan la prueba podría presentar sesgos que podrían afectar a los resultados globales del estudio.

Así, en la Tabla 5 presentamos el número de alumnos que aprobaron la prueba y el porcentaje que representa respecto al total de presentados por curso académico. Como se observa, los alumnos de primero superaron la prueba en un $88 \%$, valor muy alto, mientras que cursos superiores presentan valores inferiores, alrededor del $65 \%$.

Tabla 5. Alumnos que superan la prueba de conocimiento por curso

\begin{tabular}{|l|c|c|c|}
\hline & $\begin{array}{c}\text { Alumnos } \\
\text { presentados }\end{array}$ & $\begin{array}{c}\text { Alumnos que } \\
\text { aprueban la prueba } \\
\text { de conocimiento }\end{array}$ & $\begin{array}{c}\text { Porcentaje } \\
\text { sobre el } \\
\text { total }\end{array}$ \\
\hline Primer curso & 67 & 59 & $88 \%$ \\
\hline Segundo curso & 61 & 46 & $75 \%$ \\
\hline Tercer curso & 42 & 31 & $74 \%$ \\
\hline Cuarto curso & 26 & 16 & $62 \%$ \\
\hline Quinto curso & 25 & 17 & $68 \%$ \\
\hline Total & 221 & 169 & $76 \%$ \\
\hline
\end{tabular}

A continuación (Tabla 6), y siguiendo la tónica del apartado anterior, presentamos los estadísticos descriptivos de la media y desviación típica de la opinión que los alum- 
nos tienen de cada una de las áreas de interés presentadas: Objetivos y puesta en marcha, Estructura, Metodología, Homologación y Financiación del Plan Bolonia. Adicionalmente hemos calculado una valoración de la opinión general a través de la media del valor dado a todas las preguntas del cuestionario.

Tabla 6. Media y desviación típica de la Opinión por área y general

\begin{tabular}{|l|c|c|}
\hline & Media & Desviación Típica \\
\hline $\begin{array}{l}\text { Objetivos y puesta en } \\
\text { marcha }\end{array}$ & 3,03 &, 80 \\
\hline Estructura & 2,57 &, 81 \\
\hline Metodología & 2,92 &, 86 \\
\hline Homologación & 3,13 & 1,10 \\
\hline Financiación & 2,60 &, 92 \\
\hline Opinión general & 2,72 &, 63 \\
\hline
\end{tabular}

$\mathrm{N}=169$

Como se puede observar a raíz de la tabla, la valoración general sobre el proceso no es alta, con un valor medio de 2,72. Si vemos la valoración diferenciada en las diferentes áreas de interés, observamos que cuestiones como la homologación de títulos, los objetivos y la metodología presentan los valores más altos, mientras que la estructura y la financiación tienen los valores más bajos de la valoración. Si comparamos con la puntuación obtenida en la prueba objetiva de conocimiento, el área de financiación era la que peor nota presentaba, y ahora continua siendo de las peores valoradas. Sin embargo, los objetivos y puesta en marcha, que suspendía la prueba de conocimiento, en el cuestionario de valoración presenta la segunda puntuación más alta. Este resultado coincide con la percepción de que el estudiante de grado tiene una apreciación positiva del modo en que las metodologías docentes mejoran algunos aspectos como los contenidos teóricos y prácticos, así como las dinámicas de aula (Osoro et al., 2011). Por otra parte, este resultado no es contradictorio ya que para el análisis de la valoración o percepción hemos filtrado los alumnos que suspendían la prueba de conocimiento. En definitiva, vemos cómo la valoración negativa de un ámbito puede estar condicionada con opiniones que no están fundadas en un conocimiento real del contexto.

Por otra parte, si observamos la media general de la opinión sobre el EEES de los alumnos por curso, los alumnos de primero siguen sacando la mejor nota.

Tabla 7. Media de la Opinión por curso

\begin{tabular}{|c|c|c|c|c|}
\hline \multicolumn{5}{|c|}{ NOTA MEDIA POR CURSO } \\
\hline $\begin{array}{c}\text { Primero } \\
\text { de grado }\end{array}$ & $\begin{array}{c}\text { Segundo de } \\
\text { Licenciatura }\end{array}$ & $\begin{array}{c}\text { Tercero de } \\
\text { Licenciatura }\end{array}$ & $\begin{array}{c}\text { Cuarto de } \\
\text { Licenciatura }\end{array}$ & $\begin{array}{c}\text { Quinto de } \\
\text { Licenciatura }\end{array}$ \\
\hline 2,77 & 2,52 & 2,72 & 2,81 & 2,70 \\
\hline
\end{tabular}


Con el objeto de validar también si los resultados del cuestionario de percepción presentan diferencias significativas entre sí, de nuevo llevamos a cabo un modelo ANOVA donde la variable independiente o factor es el Curso y la variable dependiente la valoración global obtenida como la media de las respuestas a las 16 preguntas del cuestionario.

El test nos indica que existen diferencias significativas en la valoración media entre los diferentes cursos (estadístico F $=14,91$ y Sig. $=, 01$ ). Para profundizar en este apartado llevamos a cabo un análisis post hoc o comparación a posteriori. En este caso, el valor del contraste (estadístico de Levene $=27,08)$ y su nivel crítico $($ Sig. $=, 001)$ indica que no podemos asumir la igualdad de varianzas. De esta forma, hemos utilizado como contraste para establecer la comparación post hoc, la prueba T2 de Tamhane. El resultado obtenido se puede observar en la Tabla 8.

Tabla 8. Comparaciones múltiples post hoc. Prueba T2 de Tamhane

\begin{tabular}{|c|c|c|c|c|c|c|c|c|c|c|c|c|c|c|}
\hline \multicolumn{2}{|c|}{ Curso } & \multirow{2}{*}{$\begin{array}{c}\begin{array}{l}\text { Dif. de } \\
\text { medias }\end{array} \\
24^{*} \\
\end{array}$} & \multicolumn{2}{|c|}{ Curso } & \multirow{2}{*}{$\begin{array}{c}\begin{array}{c}\text { Dif. de } \\
\text { medias }\end{array} \\
-, 24^{*} \\
\end{array}$} & \multicolumn{2}{|c|}{ Curso } & $\begin{array}{l}\text { Dif. de } \\
\text { medias }\end{array}$ & \multicolumn{2}{|c|}{ Curso } & \multirow{2}{*}{$\begin{array}{c}\begin{array}{c}\text { Dif. de } \\
\text { medias }\end{array} \\
-, 04 \\
\end{array}$} & \multicolumn{2}{|c|}{ Curso } & \multirow{2}{*}{$\begin{array}{c}\begin{array}{c}\text { Dif. de } \\
\text { medias }\end{array} \\
-, 06 \\
\end{array}$} \\
\hline 1 & 2 & & 2 & 1 & & 3 & 1 &,- 04 & 4 & 1 & & 5 & 1 & \\
\hline & 3 & ,04 & & 3 &,$- 20 *$ & & 2 & ,20* & & 2 & ,28* & & 2 & ,18* \\
\hline & 4 &,- 04 & & 4 &,$- 28 *$ & & 4 &,- 08 & & 3 & ,08 & & 3 &,- 02 \\
\hline & 5 &, 06 & & 5 &,$- 18 *$ & & 5 &, 02 & & 5 &, 11 & & 4 &,- 11 \\
\hline
\end{tabular}

$* \mathrm{p}<, 05$

Esta prueba nos permite observar que a nivel de curso no hay diferencias significativas en la opinión sobre el EEES entre los alumnos de primer, tercer, cuarto y quinto curso. Sin embargo, la opinión de los alumnos de segundo curso sí difiere de forma significativa de las del resto, y con signo negativo, como se puede ver en el Gráfico 2. Este resultado al principio nos dejó un tanto sorprendidos, pero una reflexión sobre el mismo no llevó a pensar que los alumnos de segundo curso de licenciatura son realmente los más afectados por el EEES, ya que siguen sus estudios en el plan antiguo, pero el nuevo plan de estudios les puede coger en caso de fallar algún año académico, o incluso si tienen alguna asignatura suspendida de un curso a otro, ésta ya no tendría docencia, por lo que dispondrían únicamente de algunas convocatorias para superarla o se verían obligados a pasar al plan nuevo. Por otro lado, también pueden haber inquietudes vinculadas a la futura validez del título y a que sus compañeros de primer curso pueden obtener el grado en menor tiempo, tal y como también concluyen FontMayolas y Masferrer (2010). Estos hechos pensamos que son los que dan lugar a esta opinión negativa que tienen sobre el EEES. 
Gráfico 2. Gráfica de medias de opinión por curso

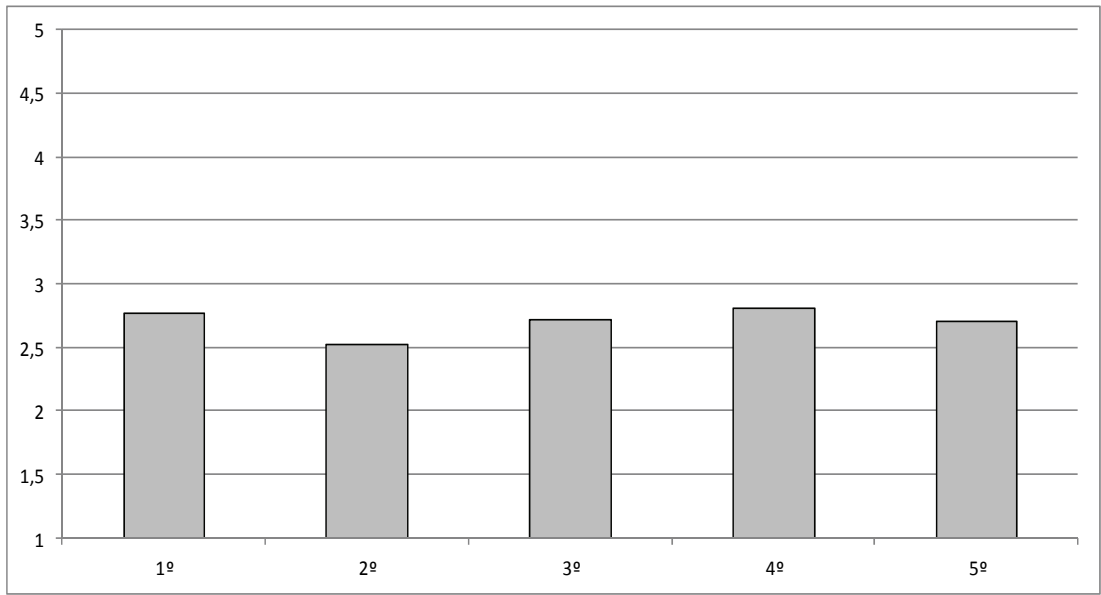

\section{Conclusiones}

La firma de la Declaración de la Sorbona instó el desarrollo de un Espacio Europeo de Educación Superior, y posteriormente la Declaración de Bolonia puso los cimientos de un largo proceso orientado a un conjunto de principios de calidad, movilidad, diversidad y competitividad que permitiera incrementar el empleo en la Unión Europea y generara un atractivo contexto universitario en Europa para que fuera un polo de atracción de estudiantes y profesores de todo el mundo. La fecha fijada como meta para la realización del EEES fue 2010, llevando en todo momento Conferencias Ministeriales de revisión y establecimiento de nuevas directrices.

A lo largo de todo este proceso ha habido todo tipo de circunstancias que han afectado a toda la comunidad universitaria de forma global. Esto ha llevado a la aparición de voces críticas, foros de discusión, manifestaciones, y como no, apoyos incondicionales al sistema, todos con argumentaciones que en la mayoría de los casos han estado reforzadas por la lógica y la reflexión, e impulsadas por las dificultades que conlleva poner en marcha un proyecto de esta ambición y envergadura.

El resultado final es que hoy Bolonia es ya una realidad, y todas las universidades están poniendo en marcha los nuevos títulos de grado. Este proceso implica un claro esfuerzo de cambio tanto por parte del profesorado como del alumnado. Por tanto, es interesante saber si este esfuerzo por parte de todos es debidamente reconocido.

En nuestro estudio empírico hemos intentado evaluar los conocimientos y la opinión que los alumnos tienen del Plan Bolonia a través de un cuestionario. En global la media resulta de "aprobado", sin embargo se pueden determinar ciertos matices interesantes. Así por ejemplo, sí hay un conocimiento sobre las nuevas metodologías, forma de evaluar, y la homologación europea de la formación superior. Por otro lado, el desconocimiento respecto al desarrollo del proceso de implantación o cómo el alumno 
puede financiar sus estudios es mayor. Adicionalmente, y como a priori cabía esperar, son los alumnos de primer curso los que poseen mayores conocimientos sobre lo que implica cursar el grado. Esto es lógico ya que ellos están viviendo en el curso actual las nuevas modalidades, y pensamos que tanto desde los centros educativos donde se imparte bachiller, orientadores, como desde la propia universidad, se ha llevado una labor intensa de información principalmente dirigida a los alumnos que se verían más afectados por el cambio. Esta conclusión vendría reforzada por los resultados significativamente más bajos obtenidos por los alumnos de cursos superiores, cuyo interés se centra principalmente en finalizar sus estudios de licenciatura.

Por otra parte, se observa disparidad entre la opinión que tienen los alumnos sobre el EEES, siendo los alumnos de segundo curso de la licenciatura (plan antiguo) los que peor valoran el plan Bolonia, mientras que los de primer curso de grado son lo que expresan mejor opinión. Este resultado consideramos que es una aportación interesante ya que el hecho de establecer un análisis comparativo entre los diferentes cursos permite ahondar un poco más en esas diferencias establecidas. Así, por ejemplo, previamente Molero López-Barajas (2007) no identificó diferencias significativas en la valoración de la docencia entre un grupo experimental de implantación del crédito europeo con otro grupo que seguía con la enseñanza del plan antiguo. Sin embargo, posteriormente $\mathrm{y}$ con el EEES en pleno funcionamiento sí observamos estas diferencias.

Este resultado es razonable en cuanto a que los alumnos de primer curso se encuentran ya inmersos en los nuevos planes de estudio. Los alumnos de cursos superiores de la licenciatura no tienen especialmente una opinión negativa. Sin embargo, la situación que en realidad más puede preocupar es la de los alumnos que están cursando segundo curso de la licenciatura (año académico 2010-2011), ya que se encuentran sometidos a cierta incertidumbre con respecto a la reforma educativa. Esto alumnos son los que más les interesa saber si van a tener que acogerse al EEES y bajo qué condiciones, ya que por ejemplo si alguna de las asignaturas de este año se les queda suspendida, deberán recuperarla ya sin docencia o desistir y cambiarse al grado.

Los resultados de este estudio sugieren implicaciones de interés, y consideramos que es deseable un mayor conocimiento del proceso para asegurar una implantación efectiva del EEES. Esta implicación coincide con el trabajo de Meroño Cerdán y Ruiz Santos (2006), donde sobre una muestra de profesores, concluyen que una mayor información dirigida a los mismos respecto al EEES mejora su actitud y su implicación futura en la reforma. En definitiva estos resultados deberían motivar a un reajuste de las estrategias de difusión y comunicación sobre el EEES (tanto en los medios utilizados, como en los contenidos y formas de presentarlos), y especialmente en las áreas con peores resultados, así como de aquellas cuestiones académicas vinculadas al proceso de implantación. Pensamos que un mayor conocimiento del proceso aseguraría una implantación mucho más efectiva del EEES, así como una opinión más favorable.

Por último, cabe apuntar que nuestro trabajo no está libre de limitaciones. En primer lugar, la propia medición mediante una batería de preguntas. Si bien se ha intentado que las pruebas sean lo más objetivas posible, en el caso de la prueba de conocimiento presenta la problemática que cualquier prueba de evaluación tiene, es decir, hasta qué punto estamos evaluando con certeza lo que conoce un alumno de una materia concre- 
ta. Por otro lado, nos hemos centrado en un perfil concreto de alumnos, por tanto, es interesante realizar el estudio en otras titulaciones, por ejemplo, de carácter más técnico, o incluso de otras universidades o centros, y establecer comparativas con los resultados obtenidos. Esta tarea la dejamos planteada como un trabajo futuro.

En definitiva, consideramos que nuestro objetivo ha sido cubierto y el resultado obtenido puede contribuir a una mejor comprensión de los cambios que el EEES está suponiendo en el mundo universitario, y principalmente desde la perspectiva del alumno. Se trata sin duda de un primer trabajo que deberemos seguir mejorando para establecer nuevas relaciones de interés que sean compartidas a través de este tipo de medios de difusión.

\section{Referencias bibliográficas}

ALBA PASTOR, C. (2005). El profesorado y las tecnologías de la información y la comunicación en el proceso de convergencia al Espacio Europeo de Educación Superior. Revista de Educación (Madrid), 337, 12-36.

DECLARACIÓN DE BOLONIA (1999). The European Higher Education Area. Declaración conjunta de los Ministros Europeos de Educación. Bolonia, 19 de junio de 1999.

DECLARACIÓN DE LA SORBONA (1998). Declaración conjunta para la armonización del diseño del sistema de educación superior europeo. Declaración a cargo de los ministros responsables de Francia, Alemania, Italia y Reino Unido. La Sorbona (París), 25 de mayo de 1998.

DÍEZ, M., PACHECO, D.I., GARCÍA, J.N., MARTÍNEZ, B., ROBLEDO, P., ÁLVAREZ, M.L., CARBONERO, M.A., ROMÁN, J.M., DEL CAÑO, M, MONJAS, I. (2009). Percepción de los estudiantes universitarios de educación respeto al uso de metodologías docentes y el desarrollo de competencias ante la adaptación al EEES: datos de la Universidad de Valladolid. Aula Abierta (Oviedo), 37 (1), 45-56.

FLORIDO DE LA NUEZ, C., JIMÉNEZ GONZÁLEZ, J.L., SANTANA MARTÍN, I. (2008). Obstáculos en el camino hacia Bolonia. Efectos de la implantación del Espacio Europeo de la Educación Superior (EEES) sobre los resultados académicos. Revista de Educación (Madrid), 354, 629-656.

FONT-MAYOLAS, S., MASFERRER, L. (2010). Conocimientos y actitudes de estudiantes universitarios respecto al Espacio Europeo de Educación Superior. Revista de Formación e Innovación Educativa Universitaria (Vigo), 3 (2), 88-96.

GARCÍA RÍOS, M.C., VALENZA, M.C., FERNÁNDEZ LAO, C., VALENZA DEMET, G., GARCÍA MARCOS, J.F. (2006). Influencia de la experiencia piloto en el conocimiento del EEES. I Jornadas de trabajo sobre experiencias piloto de implantación del crédito europeo en las Universidades Andaluzas, Cádiz, 19-21 septiembre (en papel). 
MEROÑO CERDÁN, A.L., RUÍZ SANTOS, C. (2006). Estrategias de adaptación al Espacio Europeo de Educación Superior a partir del conocimiento y actitud del profesorado universitario. Revista de Investigación Educativa (Murcia), 24 (1), 281-298.

MOLERO LÓPEZ BARAJAS, D. (2007). Rendimiento académico y opinión sobre la docencia del alumnado participante en experiencias piloto de implantación del Espacio Europeo de Educación Superior. Revista Electrónica de Investigación y Evaluación Educativa (Valencia), 13 (2).

OCAÑA, A., REYES, M.L. (2011). La voz del alumnado del grado en Maestro de Educación Infantil en la Universidad de Granada: una evaluación cualitativa a través del grupo de discusión. Revista Interuniversitaria de Formación del Profesorado (Zaragoza), 14 (4).

OSORO, J.M., ARGOS, J., SALVADOR, L., EZQUERRA, P., CASTRO, A. (2011). La implantación de las titulaciones de Grado de los estudios de Educación: algunas reflexiones y constataciones desde las miradas de docentes y estudiantes. Revista Interuniversitaria de Formación del Profesorado (Zaragoza), 14 (4). 
Anexo I

Prueba objetiva de conocimiento sobre el Plan Bolonia

1-. ¿Cuáles son los objetivos del Espacio Europeo de Educación Superior (EEES)?

-Movilidad y Adaptabilidad

-Adaptabilidad y Empleabilidad

-Movilidad y Empleabilidad

2-. ¿Cómo se divide el nuevo plan de estudios?

-Grado y Máster

-Grado y Posgrado

-Ingeniería y Doctorado

3-. ¿A su vez, qué dos etapas conforman la segunda parte del plan de estudios anteriormente citado?

-Curso y Máster

-Máster y Doctorado

-Curso y Doctorado

4-. ¿Cómo se llama el nuevo título de fin de estudios?

-Suplemento europeo al título

-Título fin de carrera

-Suplemento final de estudios

5-. ¿Cuál es el plazo máximo de adaptación de las titulaciones al Espacio Europeo de Educación Superior?

$-2012$

$-2010 / 2011$

$-2015$ 
6-- ¿Qué método de evaluación se va a utilizar principalmente en el EEES?

-Evaluación final

-Evaluación por puntos

-Evaluación continua

7-. ¿Cómo se llaman los nuevos créditos docentes?

-Créditos CEM

-Créditos ECTS

-Créditos europeos

8-. ¿De cuantos créditos consta cada titulación de grado?

-240 créditos

-300 créditos

-450 créditos

9-. ¿Qué facilitará el Plan Bolonia con sus nuevos créditos?

-La equivalencia entre estudios universitarios en toda Europa

- Valorar tu nivel de estudios en cualquier momento

-Valorar tu capacidad intelectual en toda Europa

10-. ¿Sabes cuál es el nuevo método de financiación establecido por el Plan Bolonia para costear los estudios, $y$ en qué consiste?

-Financiación bancaria $\rightarrow$ financiación de la matrícula universitaria sin intereses

-Préstamo renta-universidad $\rightarrow$ renta mensual + ayuda mensual complementaria -Pago aplazado $\rightarrow$ pago a plazos del coste del curso académico universitario 


\section{$\underline{\text { Cuestionario de percepción sobre el Plan Bolonia }}$}

[Valorar del 1 al 5 siendo 1=Totalmente en desacuerdo, 2=En desacuerdo, 3 =Término medio, $4=$ De acuerdo, $5=$ Totalmente de acuerdo]

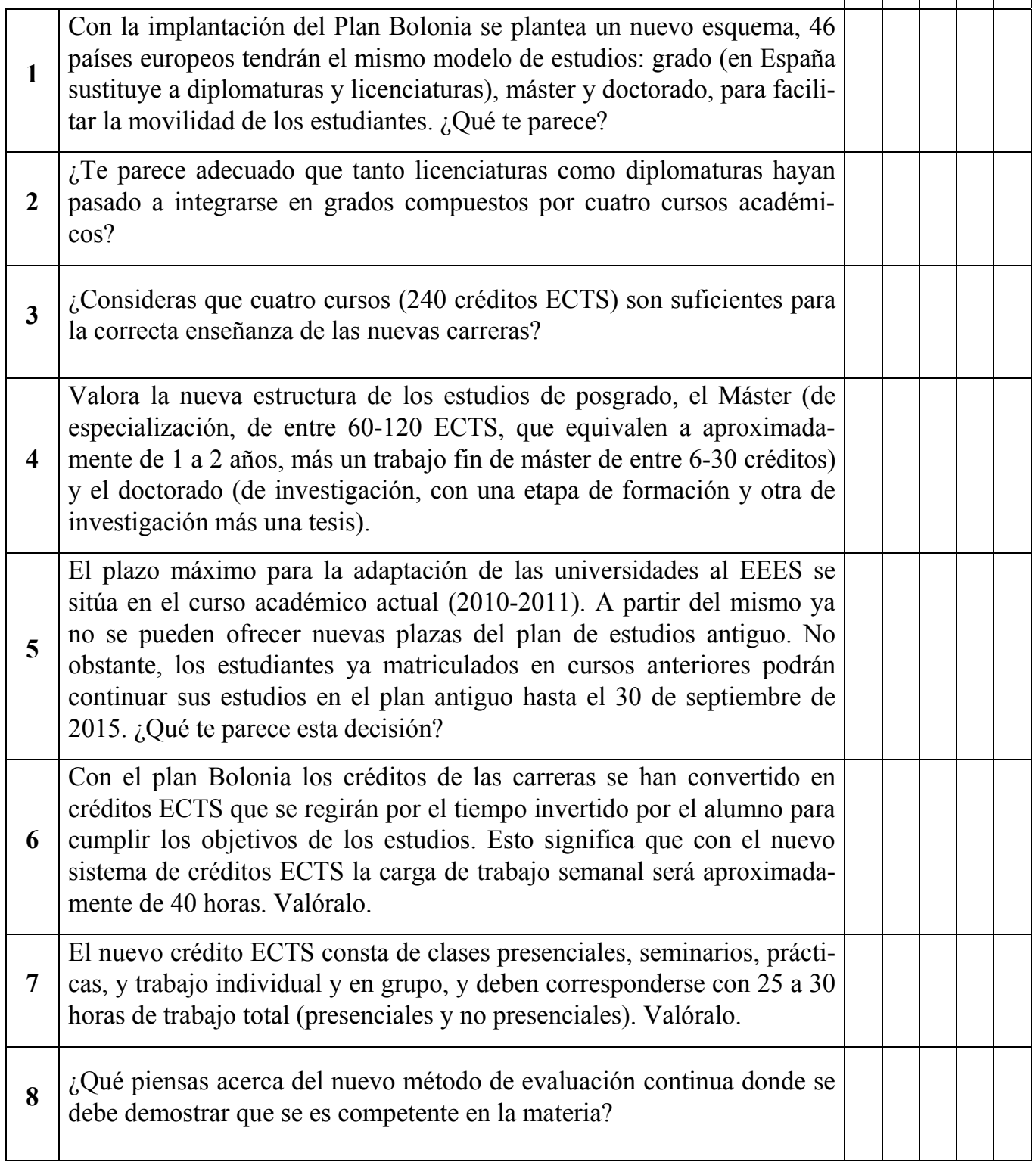




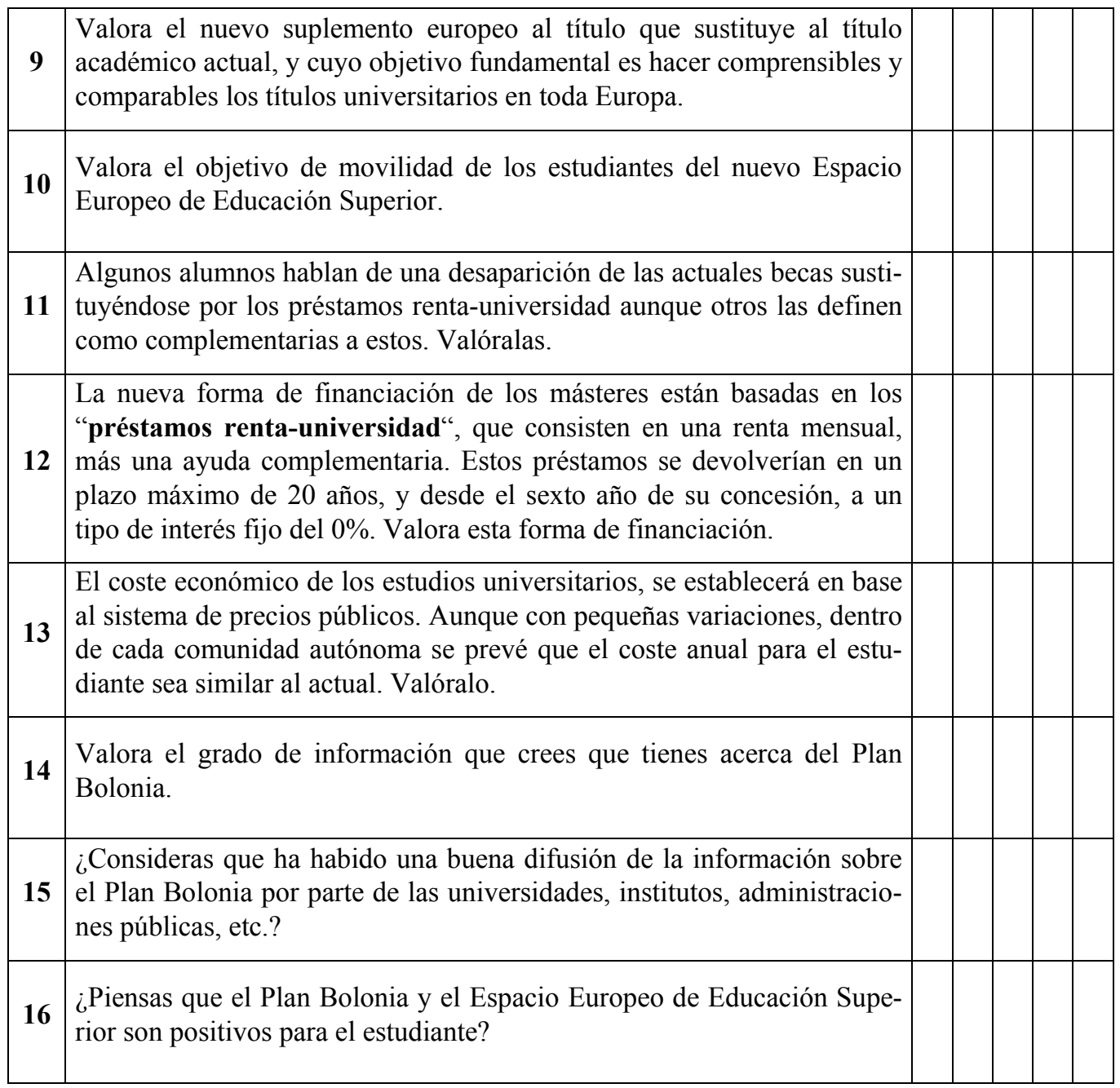




\section{Correspondencia con los autores}

Manuel Expósito-Langa

Universitat Politècnica de València

Departamento de Organización de Empresas

Plaza Ferrándiz y Carbonell 2

03801. Alcoy (Alicante)

Tlf.: 966528466

E-mail:maexlan@doe.upv.es

José-Vicente Tomás-Miquel

Universitat Politècnica de València

Departamento de Organización de Empresas

Plaza Ferrándiz y Carbonell 2

03801. Alcoy (Alicante)

Tlf.: 966528466

E-mail: jotomi@doe.upv.es

Gema Torres Gallardo

Universitat Politècnica de València

Plaza Ferrándiz y Carbonell 2

03801. Alcoy (Alicante)

E-mail: getorgal@epsa.upv.es 